\title{
PARTITIONS, EGYPTIAN FRACTIONS, AND FREE PRODUCTS OF FINITE ABELIAN GROUPS
}

\author{
MICHAEL ANSHEL AND DORIAN GOLDFELD
}

(Communicated by Andrew Odlyzko)

\begin{abstract}
Some computational questions concerning free products of finite abelian groups are reduced to questions in additive number theory involving partitions and Egyptian fractions. These in turn are resolved employing the Mathematica ${ }^{T M}$ computer system.
\end{abstract}

\section{INTRODUCTION}

In [2], it is shown that if

$$
G=G_{1} * G_{2} * \cdots * G_{r}
$$

is the free product of $r \geq 2$ finite abelian groups $G_{i}$ of order $q_{i} \geq 2$, then the commutator subgroup $G^{\prime}$ of $G$ is a free group of rank $s$ where

$$
s=r q-q\left(\sum_{i=1}^{r} \frac{1}{q_{i}}\right)-(q-1)
$$

and $q=q_{1} q_{2} \cdots q_{r}$ is the index of $G^{\prime}$ in $G$. Without loss of generality we may assume $q_{1} \leq q_{2} \leq \cdots \leq q_{r}$. We refer to $\left(q_{1}, q_{2}, \ldots, q_{r}\right)$ as an order type of $G$.

The following basic question provoked our investigation:

To what extent does the index $q$, the number of factors $r$, and

the commutator subgroup $G^{\prime}$ of $G$ (given by (1) above) uniquely

determine the order type $\left(q_{1}, q_{2}, \ldots, q_{r}\right)$ ?

For given positive integers $q, r, s$, with $r>2$, let $\Gamma(q, r, s)$ denote the class of all groups $G$ which are free products of $r$ finite abelian groups and whose commutator subgroup $G^{\prime}$ satisfies

$$
\left[G: G^{\prime}\right]=q, \quad \operatorname{rank}\left(G^{\prime}\right)=s,
$$

Received by the editors July 28, 1989 and, in revised form, April 2, 1990.

1980 Mathematics Subject Classification (1985 Revision). Primary 11A67, 11P57, 20 E06.

Key words and phrases. Partitions, Egyptian fractions, linear diophantine problems, free products of finite abelian groups, commutator subgroups.

Supported in part by NSF grant no. DMS-87-02169. 
and let $\Gamma(q, r)=\bigcup_{s} \Gamma(q, r, s)$. Let $\mathrm{C}(q, r, s)$ denote the number of distinct order types associated with the groups of $\Gamma(q, r, s)$. For fixed $q, r$ question (3) is equivalent to asking for which values of $s \in \mathbb{Z}$ is $C(q, r, s)=1$. Accordingly, let $C(q, r)$ denote the number of $s \in \mathbb{Z}$ for which

$$
C(q, r, s)>1 \text {. }
$$

We shall completely determine $C(q, r)$ in the special case that $q$ is a prime power and $r$ is small. This answers (3) for this case. If $q$ is a prime power and $r$ is large, we reduce (3) to a linear diophantine problem and provide an effective algorithm for computing $C(q, r)$.

It is known [4] that the number of nonisomorphic abelian groups of order $m=\prod_{1}^{k} l_{i}^{e_{i}}$ (here $l_{i}$ denote the distinct primes dividing $m$ ) is given by

$$
N(m)=p\left(e_{1}\right) p\left(e_{2}\right) \cdots p\left(e_{k}\right)
$$

where $p$ is the partition function. It follows that if $\{q, r, s\}$ uniquely determines the order type $\left(q_{1}, q_{2}, \ldots, q_{r}\right)$ then the number of factorizations defining groups in $\Gamma(q, r, s)$ is precisely $\prod_{i=1}^{r} N\left(q_{i}\right)$. It is a consequence of the Kurosh isomorphism theorem [5, p. 245] that distinct factorizations of type (1) define nonisomorphic groups.

We now reformulate the question (3) as a problem concerning partitions and Egyptian fractions. Following Schroeder [6], an Egyptian fraction is a unit fraction, i.e. a rational number with numerator equal to one. Traditionally, an Egyptian fraction expansion is the representation of a rational number as a sum of distinct unit fractions (see [3]). Note that for the Egyptians, the basic fractions were the unit fractions and the special fraction $\frac{2}{3}$. The Rhind papyrus (2000-1800 B.C.) gives an algorithm for representing rational numbers as sums of these basic fractions [7]. We generalize the traditional concept of expansion and define an Egyptian fraction decomposition as the representation of a rational number as a sum of unit fractions which may not necessarily be distinct.

Let $q$ be a positive integer. For $\alpha \in \mathbb{Q}, r=2,3, \ldots$ let $M(q, r, \alpha)$ denote the number of $r$-tuples of integers $\left\{q_{1}, q_{2}, \ldots, q_{r}\right\}$, satisfying

$$
\begin{aligned}
& \text { (a) } 2 \leq q_{1} \leq q_{2} \cdots \leq q_{r}, \\
& \text { (b) } q=q_{1} q_{2} \cdots q_{r} \quad \text { (multiplicative condition), } \\
& \text { (c) } \alpha=\sum_{i=1}^{r} \frac{1}{q_{i}} \quad \text { (Egyptian fraction decomposition), }
\end{aligned}
$$

and let $M(q, r)$ denote the number of $\alpha \in \mathbb{Q}$ for which $M(q, r, \alpha)>1$. The condition $M(q, r)=0$ is equivalent to the fact that for each $\alpha \in \mathbb{Q}$, if an $r$-tuple $\left\{q_{1}, q_{2}, \ldots, q_{r}\right\}$ satisfying (5) exists, then it must be unique.

It is easily checked that

$$
M(q, r, r-1+(1-s) / q)=C(q, r, s)
$$

and

$$
M(q, r)=C(q, r),
$$

and this provides the required reformulation. 
We shall show that the computation of $M(q, r)$ is equivalent to a certain linear diophantine problem in the special case that $q$ is a power of a prime $l$. Our main results are:

Theorem 1. Let $q=l^{n}$ be a prime power. If $r<2 l+1$ then $M(q, r)=0$; hence any rational number $\alpha$ has at most one Egyptian fraction decomposition of type (5). If $r=2 l+1$ then $M(q, r)$ is precisely the number of integer solutions $\{a, b\}$ satisfying

$$
\begin{aligned}
& 1 \leq a<b<2 b-a+1, \\
& n=(2 l+1) b+3 l .
\end{aligned}
$$

Moreover, if $r=2 l+1$ then $M(q, r, \alpha) \leq 2$ for all rational $\alpha$.

Theorem 2. Let $q=l^{n}$ be a prime power. If $2<r \leq 2 l+1$ then the number of nonisomorphic commutator subgroups associated to groups in $\Gamma(q, r)$ is

$$
\sum_{\substack{e_{1}+e_{2}+\cdots+e_{r}=n \\ 1 \leq e_{1} \leq \cdots \leq e_{r}}} 1,
$$

the number of integer partitions of $n$ into $r$ positive parts, unless $r=2 l+1, n=$ $3 l+b(2 l+1)$ with $b=2,3,4, \ldots$. In this case, the number of nonisomorphic commutator subgroups is given by

$$
\sum_{\substack{e_{1}+e_{2}+\cdots+e_{r}=n \\ 1 \leq e_{1} \leq \cdots \leq e_{r}}} 1-(b-1) .
$$

Let $\mathscr{A}$ be a class of finite abelian groups. We say $\mathscr{A}$ is order determined provided $G, H \in \mathscr{A}$ are isomorphic if and only if $|G|=|H|$ (e.g. the finite cyclic groups). Let $\Gamma_{\mathscr{A}}(q, r)$ denote those groups $G \in \Gamma(q, r)$ whose factors $G_{i} \in \mathscr{A}$.

As a consequence of Theorem 1, we shall obtain:

Corollary 3. Let $\mathscr{A}$ be a class of finite abelian groups which is order determined and possesses groups of every possible order and let $q=l^{n}$ be a prime power. If $r<2 l+1$ or $r=2 l+1$ and $n \not \equiv 3 l(\bmod 2 l+1)$, then $G, H \in \Gamma_{\mathscr{A}}(q, r)$ are isomorphic if and only if their commutator subgroups $G^{\prime}, H^{\prime}$ are isomorphic.

Remarks. If $q=l^{n}$ is a prime power, our methods yield an algorithm for determining $\mathrm{C}(q, r)$ for any fixed $r>2 l+1$. In general, this algorithm reduces the computation of $\mathrm{C}(q, r)$ to counting the number of solutions of a certain linear diophantine problem, of the type considered in [1].

To illustrate Theorem 1 we consider the case where $l=2, b=2, r=5, n=$ 16. We have $M\left(2^{16}, 5\right)=1$ and the associated decompositions are

$$
\frac{1}{2}+\frac{1}{8}+\frac{1}{16}+\frac{1}{16}+\frac{1}{16}=\frac{1}{4}+\frac{1}{4}+\frac{1}{4}+\frac{1}{32}+\frac{1}{32}=\frac{13}{16} .
$$

Similarly, when $l=2, b=3, r=5, n=21$, we have $M\left(2^{21}, 5\right)=2$. The associated decompositions are

$$
\frac{1}{4}+\frac{1}{16}+\frac{1}{32}+\frac{1}{32}+\frac{1}{32}=\frac{1}{8}+\frac{1}{8}+\frac{1}{8}+\frac{1}{64}+\frac{1}{64}=\frac{13}{32},
$$




$$
\frac{1}{2}+\frac{1}{16}+\frac{1}{32}+\frac{1}{32}+\frac{1}{64}=\frac{1}{4}+\frac{1}{4}+\frac{1}{8}+\frac{1}{128}+\frac{1}{128}=\frac{41}{64} \text {. }
$$

If $q$ is not a prime power, then the situation becomes considerably more complex. Nevertheless, if $r=2$ we will always have $M(q, 2)=0$. This is because the equations

$$
\begin{aligned}
q_{1} q_{2} & =q, \\
\frac{1}{q_{1}}+\frac{1}{q_{2}} & =\alpha
\end{aligned}
$$

either have no solutions in integers $q_{1}, q_{2}$, or else must have a unique solution, $2 \leq q_{1} \leq q_{2}$, in integers $q_{1}, q_{2}$. On the other hand, if $r=3$, it is not hard to find $q$ for which $M(q, r) \geq 1$. This occurs, for example, if $r=3$ and $q=600$ since

$$
\begin{gathered}
\frac{5}{12}=\frac{1}{4}+\frac{1}{10}+\frac{1}{15}=\frac{1}{5}+\frac{1}{6}+\frac{1}{20}, \\
4 \cdot 10 \cdot 15=5 \cdot 6 \cdot 20=600
\end{gathered}
$$

and

$$
\begin{gathered}
\frac{29}{60}=\frac{1}{3}+\frac{1}{10}+\frac{1}{20}=\frac{1}{4}+\frac{1}{5}+\frac{1}{30}, \\
3 \cdot 10 \cdot 20=4 \cdot 5 \cdot 30=600 .
\end{gathered}
$$

A more interesting example is furnished by $r=3, q=2 \cdot 3 \cdot 5 \cdot 7 \cdot 11 \cdot 13 \cdot 17$. In this example

$$
\frac{35963}{510510}=\frac{1}{17}+\frac{1}{154}+\frac{1}{195}=\frac{1}{26}+\frac{1}{33}+\frac{1}{595}
$$

and

$$
17 \cdot 154 \cdot 195=26 \cdot 33 \cdot 595=510510=q .
$$

\section{Proof of Theorems 1 AND 2}

Let $l$ be a prime number, and let

$$
\left[a_{1}, a_{2}, \ldots, a_{m}\right]=\sum_{i=1}^{m} l^{-a_{i}}
$$

represent a length $m$ Egyptian fraction decomposition of $\alpha=\sum_{i=1}^{m} l^{-a_{i}}$ (with respect to $l)$. We let $E_{m}(\alpha)$ denote the set of all such length $m$ decompositions. Similarly, define

$$
E_{m}^{*}(\alpha)=\left\{\left[a_{1}, a_{2}, \ldots, a_{m}\right] \in E_{m}(\alpha) \mid a_{1} \leq a_{2} \leq \cdots \leq a_{m}\right\} .
$$

Since

$$
l^{-a}=\underbrace{l^{-a-1}+\cdots+l^{-a-1}}_{l \text { times }}
$$

we see that

$$
\left[a_{1}, a_{2}, \ldots, a_{m}\right]=[a_{1}, \ldots, a_{i-1}, \overbrace{a_{i}+1, \ldots, a_{i}+1}^{l \text { times }} a_{i+1}, \ldots, a_{m}] .
$$


It is, therefore, natural to define an expansion operator

$$
T_{i}: E_{m}(\alpha) \longrightarrow E_{m+l-1}(\alpha)
$$

where

$$
T_{i}\left(\left[a_{1}, a_{2}, \ldots, a_{m}\right]\right)=[a_{1}, \ldots, a_{i-1}, \overbrace{a_{i}+1, \ldots, a_{i}+1}^{l \text { times }} a_{i+1}, \ldots, a_{m}] .
$$

This operator may be extended to an operator

$$
T: E_{m}(\alpha) \longrightarrow \operatorname{Pow}\left(E_{m+l-1}(\alpha)\right)
$$

where for $A \in E_{m}(\alpha)$,

$$
T(A)=\bigcup_{i=1}^{m} T_{i}(A)
$$

We can further extend $T$ to an operator on subsets

$$
T: \operatorname{Pow}\left(E_{m}(\alpha)\right) \longrightarrow \operatorname{Pow}\left(E_{m+l-1}(\alpha)\right)
$$

by requiring that for $X \in \operatorname{Pow}\left(E_{m}(\alpha)\right)$

$$
T(X)=\bigcup_{\substack{A \in X \\ A \in E_{m}(\alpha)}} T(A) .
$$

If $0<\alpha<l$ is a rational number given by a reduced fraction whose denominator is of the form $l^{m}$, then $\alpha$ has a unique $l$-adic expansion

$$
\alpha=\sum_{\substack{i=1 \\ 0 \leq a_{1}<a_{2}<\cdots<a_{m}}}^{m} c\left(a_{i}\right) l^{-a_{i}}
$$

where $1 \leq c\left(a_{i}\right) \leq l-1$ for $1 \leq i \leq m$. Set $c=\sum_{i=1}^{m} c\left(a_{i}\right)$. This data determines a unique element $A_{\alpha} \in E_{c}^{*}(\alpha)$ given by

$$
A_{\alpha}=\left[\operatorname{rep}\left(a_{1}, c\left(a_{1}\right)\right), \operatorname{rep}\left(a_{2}, c\left(a_{2}\right)\right), \ldots, \operatorname{rep}\left(a_{m}, c\left(a_{m}\right)\right)\right]
$$

where

$$
\operatorname{rep}(a, t)=\underbrace{a, a, \ldots, a}_{t \text { times }} .
$$


We now prove the fundamental lemma:

Lemma 4. Let $0<\alpha<l$ be a rational number given by a reduced fraction whose denominator is of the form $l^{m}$, and let $A_{\alpha}$ be as above. Then any Egyptian fraction decomposition of $\alpha$

$$
\left[a_{1}, a_{2}, \ldots, a_{n}\right]
$$

satisfies

$$
\left[a_{1}, a_{2}, \ldots, a_{n}\right] \in T^{k}\left(A_{\alpha}\right)
$$

for some integer $k$ which depends on $n$ and l. Hence

$$
E_{n}(\alpha) \subset T^{k}\left(A_{\alpha}\right) \text {. }
$$

Proof of Lemma 4. We define an ordering operator Ord : $E_{n}(\alpha) \rightarrow E_{n}^{*}(\alpha)$ as follows:

$$
\operatorname{Ord}\left(\left[a_{1}, a_{2}, \ldots, a_{n}\right]\right)=\left[a_{1}^{\prime}, a_{2}^{\prime}, \ldots, a_{n}^{\prime}\right]
$$

where

$$
\left\{a_{1}, a_{2}, \ldots, a_{n}\right\}=\left\{a_{1}^{\prime}, a_{2}^{\prime}, \ldots, a_{n}^{\prime}\right\}
$$

as sets and

$$
a_{1}^{\prime} \leq a_{2}^{\prime} \leq \cdots \leq a_{n}^{\prime} .
$$

Let $A=\left[a_{1}, a_{2}, \ldots, a_{n}\right] \in E_{n}(\alpha)$. Without loss of generality we may assume that $a_{1} \leq a_{2} \leq \cdots \leq a_{n}$. By abuse of notation, we also consider $A$ as a set $\left\{a_{1}, a_{2}, \ldots, a_{n}\right\}$. If $l$ elements of $A$ are equal to $a_{j}$, say,

$$
a_{j}=a_{j+1}=\cdots=a_{j+l-1},
$$

then we call

$$
\left\{a_{j}, a_{j+1}, \ldots, a_{j+l-1}\right\}
$$

an $l$-block of $a_{j}$ 's. If such an $l$-block occurs then we may define a contraction operator $\hat{T}_{j}$ by

$$
\begin{aligned}
& \hat{T}_{j}\left([a_{1}, \ldots, a_{j-1}, \overbrace{a_{j}, \ldots, a_{j+l-1}}^{l \text {-block }}, a_{j+l}, \ldots, a_{n}]\right) \\
& \quad=\left[a_{1}, \ldots, a_{j-1}, a_{j}-1, a_{j+l}, \ldots, a_{n}\right]
\end{aligned}
$$

which replaces the $l$-block

$$
\left\{a_{j}, a_{j+1}, \ldots, a_{j+l-1}\right\}
$$

by the single element $a_{j}-1$.

We now prove Lemma 4. The assumption $0<\alpha<l$ ensures that

$$
0 \leq a_{1} \leq a_{2} \leq \cdots \leq a_{n} .
$$

Now recursively contract and reorder, that is recursively apply the contraction operator

$$
\operatorname{Ord} \circ \hat{T}_{j}
$$


to

$$
\left[a_{1}, a_{2}, \ldots, a_{n}\right]
$$

whenever some $l$-block of $a_{j}$ 's occurs. After finitely many contractions depending on $n$ and $l$ we obtain a new Egyptian fraction decomposition

$$
B=\left[b_{1}, b_{2}, \ldots, b_{s}\right] \in E_{s}(\alpha)
$$

where $s \leq n$ and no value $b_{j}$ is repeated $l$ or more times. It follows that

$$
B=A_{\alpha}
$$

must be the unique $l$-adic expansion of $\alpha$. By reversing all steps, it is clear that if $B=A_{\alpha}$ is obtained after $k$ contractions of $A$ then

$$
\left[a_{1}, a_{2}, \ldots, a_{n}\right] \in T^{k}\left(E\left(A_{\alpha}\right)\right) .
$$

This completes the proof.

For $A=\left[a_{1}, \ldots, a_{m}\right] \in E_{m}(\alpha)$, we define

$$
\begin{aligned}
& S(A)=\sum_{i=1}^{m} a_{i}, \\
& L(A)=m .
\end{aligned}
$$

We now prove Theorem 1 . Let $q=l^{n}$ and $r \leq 2 l+1$. Fix $\alpha \in \mathbb{Q}$. It follows from (5c) and the conditions $r \leq 2 l+1, n \geq 1$ that $\alpha \leq 2+1 / l$. Assume (5) has two solutions $\left\{q_{1}, q_{2}, \ldots, \bar{q}_{r}\right\}$ and $\left\{q_{1}^{\prime}, q_{2}^{\prime}, \ldots, q_{r}^{\prime}\right\}$ where

$$
q_{i}=l^{a_{i}} \text { and } q_{i}^{\prime}=l^{a_{i}^{\prime}}
$$

for $1 \leq i \leq r$. This gives rise to two sets

$$
U=\left[a_{1}, a_{2}, \ldots, a_{r}\right] \text { and } V=\left[a_{1}^{\prime}, a_{2}^{\prime}, \ldots, a_{r}^{\prime}\right]
$$

in $E_{r}(\alpha)$. It follows that we must have

$$
U, V \in T^{\mu}\left(A_{\alpha}\right)
$$

where $0 \leq \mu \leq 3$ since $r \leq 2 l+1$. Moreover the condition

$$
\prod_{i=1}^{r} q_{i}=\prod_{i=1}^{r} q_{i}^{\prime}=q
$$

implies that $S(U)=S(V)$.

We have used a Macintosh II computer with the computer language Mathematica $^{T M}$ [8] to find all solutions in integers $\mu$ and Egyptian fraction decompositions

$$
U, V, A \in \bigcup_{\alpha \in \mathbb{Q}} \bigcup_{m=1}^{\infty} E_{m}(\alpha)
$$


to the problem

$$
\begin{gathered}
U, V \in T^{\mu}(A) \quad(0 \leq \mu \leq 3), \\
S(U)=S(V), \\
U \neq V(\text { as sets. }), \\
L(U)=L(V) \leq 2 l+1 .
\end{gathered}
$$

The only solution to (7) found was

$$
\begin{aligned}
A & =[a, b, c] \quad \text { with } \quad 1 \leq a<b<c, \\
U & =[\overbrace{a+1, \ldots, a+1}^{l \text { times }}, b, \overbrace{c+1, \ldots, c+1}^{l \text { times }}], \\
V & =[a, \overbrace{b+1, \ldots, b+1}^{(l-1) \text { times }}, \overbrace{b+2, \ldots, b+2}^{l \text { times }}, c] .
\end{aligned}
$$

Here $S(U)=l a+b+l c+2 l . \quad S(V)=a+(2 l-1) b+c+3 l-1$, and the condition $S(U)=S(V)$ implies that $c=2 b-a+1$. Since $S(U)=S(V)=n$, we must also have $n=a+(2 l-1) b+c+c l-1$. These results imply that if $r<2 l+1$ then $M\left(l^{n}, r\right)=0$, while if $r=2 l+1$, then

$M\left(l^{n}, r\right)=\operatorname{Card}\{[a, b, c] \mid 1 \leq a<b<c, c=2 b-a+1, n=(2 l+1) b+3 l\}$

which is precisely the formula given in Theorem 1 .

We now briefly describe the algorithm for solving (7). First, if the length of $A$ is greater than three then we must have $U, V \in T(A)$. It is easily checked that there can be no solutions to (7) in this case. If the length of $A$ is three, set

$$
A=[a, b, c] \text {, }
$$

with $a \leq b \leq c$. Then $T(A)$ consists of the following 3 elements:

$$
\begin{aligned}
& {[\operatorname{rep}(a+1, l), b, c],} \\
& {[a, \operatorname{rep}(b+1, l), c],} \\
& {[a, b, \operatorname{rep}(c+1, l)] .}
\end{aligned}
$$

One immediately checks that there can be no solutions to (7) among the above 3 elements. Similarly $T^{2}(A)$ consists of the following elements:

$$
\begin{gathered}
{[\operatorname{rep}(a+1, l-1), \operatorname{rep}(a+2, l), b, c],} \\
{[\operatorname{rep}(a+1, l), \operatorname{rep}(b+1, l), c],} \\
{[\operatorname{rep}(a+1, l), b, \operatorname{rep}(c+1, l)],} \\
{[\operatorname{rep}(a+1, l), \operatorname{rep}(b+1, l), c],} \\
{[a, \operatorname{rep}(b+1, l-1), \operatorname{rep}(b+2, l), c],} \\
{[a, \operatorname{rep}(b+1, l), \operatorname{rep}(c+1, l)],}
\end{gathered}
$$




$$
\begin{gathered}
{[\operatorname{rep}(a+1, l), b, \operatorname{rep}(c+1, l)],} \\
{[a, \operatorname{rep}(b+1, l), \operatorname{rep}(c+1, l)],} \\
{[a, b, \operatorname{rep}(c+1, l-1), \operatorname{rep}(c+2, l)] .}
\end{gathered}
$$

By examining all eighty-one pairs of the above elements, it is shown that the only solutions to (7) of the above type are given by (8). The calculations for $A$ of lengths one and two are entirely similar and lead to no additional solutions of (7).

To prove Theorem 2, note that from (2) the number of nonisomorphic commutator subgroups associated to groups in $\Gamma(q, r)$ is just

$$
\sum_{\substack{q_{1} \leq q_{2} \leq \cdots \leq q_{r} \\ q_{1} \cdots q_{r}=q}} 1-C(q, r)
$$

since in this case $C(q, r, s) \leq 2$ for all ranks $s \in \mathbb{Z}$, by Theorem 1 and (6). But setting $q_{i}=l^{e_{i}}$ and $q=l^{n}$, we have

$$
\sum_{\substack{q_{1} \leq q_{2} \leq \cdots \leq q_{r} \\ q_{1} \cdots q_{r}=q}} 1=\sum_{\substack{e_{1}+\cdots+e_{r}=n \\ 1 \leq e_{1} \leq \cdots \leq e_{r}}} 1 .
$$

Moreover, $C(q, r)=M(q, r)$, and by Theorem $1, M(q, r)=0$ unless $n=$ $(2 l+1) b+3 l$, in which case $M(q, r)=b-1$.

\section{ANother Proof of Theorem 1}

The referee has kindly allowed us to include an alternative proof of Theorem 1 which was discovered while reviewing this paper.

Suppose $\left\{p_{1}, \ldots, p_{r}\right\}$ and $\left\{q_{1}, \ldots, q_{r}\right\}$ are given in increasing order, and suppose $\Pi p_{i}=\prod q_{i}=l^{n}$, where $l \geq 2$ is prime, and $\alpha=\sum p_{i}^{-1}=\sum q_{i}^{-1}$. Then $p_{i}=l^{a_{i}}$ and $q_{i}=l^{b_{i}}, a_{i}, b_{i} \geq 1$ and $\sum a_{i}=\sum b_{i}=n$. Let $c_{j}$ (resp. $d_{j}$ ) denote the number of $a_{i}$ 's (resp. $b_{i}$ 's) which equal $j$. Then $\left\{c_{j}\right\}$ and $\left\{d_{j}\right\}$ satisfy the following conditions:

$$
\begin{aligned}
\sum c_{j} & =\sum d_{j}=r, \\
\sum j c_{j} & =\sum j d_{j}=n, \\
\sum c_{j} l^{-j} & =\sum d_{j} l^{-j}=\alpha .
\end{aligned}
$$

Conversely, any choice of $\left\{c_{j}\right\}$ and $\left\{d_{j}\right\}$ satisfying (i) through (ii) will satisfy the conditions outlined in the first sentences. Let $e_{j}=c_{j}-d_{j}$ and let $P(x)$ denote the polynomial $\sum\left(c_{j}-d_{j}\right) x^{j}$. Then (i) through (iii) imply that $P(1)=$ $P^{\prime}(1)=P\left(l^{-1}\right)=0$ so that, as real polynomials,

$$
P(x)=\sum e_{j} x^{j}=(1-x)^{2} \cdot(1-l x) \cdot\left(\sum f_{j} x^{j}\right) .
$$


Since the $e_{j}$ 's are integers and the factor has leading unit terms, it is easy to show by induction that the $f_{j}$ 's are also integers: (9) gives the complete parametrization of $\left\{p_{i}\right\}$ and $\left\{q_{i}\right\}$ satisfying the conditions of the problem.

We are interested in the case $r \leq 2 l+1$. Since $\left|e_{j}\right|=\left|c_{j}-d_{j}\right| \leq c_{j}+d_{j}$, this implies that $\|P\|:=\sum\left|e_{j}\right| \leq 2 r \leq 4 l+2$. One possible direction is to observe from (9) that $|P(-1)|=\left|\sum(-1)^{j} e_{j}\right| \leq \sum e_{j}$ and $P(-1)=4(l+1) \sum(-1)^{j} f_{j}$, hence $\sum(-1)^{j} f_{j}=0$; that is, $(x+1) \mid P(x)$.

It is actually easier to backtrack somewhat. Write

$$
P(x)=\sum e_{j} x^{j}=(1-l x) \cdot \sum g_{j} x^{j},
$$

where each $g_{j}$ is an integer, and further, $\sum g_{j}=\sum j g_{j}=0$ (in view of (9)). Write $\sum g_{j} x^{j}$ as a sum (the same number of) $x^{t_{i}}$, s and $-x^{u_{j}}$, s:

$$
\sum g_{j} x^{j}=x^{t_{1}}+\cdots+x^{t_{s}}-x^{u_{j}}-\cdots-x^{u_{s}} \quad\left(t_{1} \leq \cdots \leq t_{s}, u_{1} \leq \cdots \leq u_{s}\right) .
$$

There may be some repeats among the $t$ 's and among the $u$ 's if $\left|g_{j}\right| \geq 2$, but no $t_{i}$ is also a $u_{j}$. Further, $\sum t_{i}=\sum u_{j}$ because $\sum j g_{j}=0$. It follows that

$$
P(x)=x^{t_{1}}-l x^{t_{1}+1}+\cdots+x^{t_{s}}-l x^{t_{s}+1}-x^{u_{1}}+l x^{u_{1}+1}+\cdots-x^{u_{s}}+l x^{u_{s}+1} .
$$

What is $\|P\|$, the sum of the absolute values of the coefficients of $P(x)$ ? It is $2 s(l+1)$ minus whatever cancellation occurs. Observe that the $x^{t_{j}}$ 's and $x^{t_{i}+1}$, s cannot cancel with any $x^{u_{j}}$ or $x^{u_{j}+1}$, because no $t_{i}$ is a $u_{j}$. If any power of $x$ repeats, it does so with the same sign, and no cancellation occurs. Any cancellation occurs among the $t_{i}$ 's and among the $u_{j}$ 's; since $x^{t_{i}}$ and $x^{u_{1}}$ can't cancel, having minimal exponent, at most $s-1$ powers of $x$ are cancelled among the $t$ 's and among the $u$ 's, so $\|P\| \geq 2 s(l+1)-4(s-1)$. Thus, $4 l+2 \geq 2 s(l+1)-4(s-1)=2 s(l-1)+4$. It follows that $(2 l-1) /(l-1) \geq s$. If $l \geq 3$, this implies that $s=1$ or 2 . If $l=2$, then $s=1,2$ or 3 ; but if $s=3$, then the maximal cancellation occurs.

If $s=1$, then $\sum t_{j}=\sum u_{j}$ implies that $t_{1}=u_{1}$, which is excluded. If $s=2$, then $t_{1}+t_{2}=u_{1}+u_{2}$. Assume without loss of generality that $a=t_{1} \leq$ $u_{1}=b$ and that $u_{2}=b+d$. Then $t_{2}=c=2 b+d-a \geq u_{2}$ and

$$
P(x)=x^{a}-l x^{a+1}-x^{b}+l x^{b+d}+l x^{b+d+1}-x^{b+d}+l x^{b+d+1}+x^{c}-l x^{c+1} .
$$

The only opportunity to cancel in $\|P\|$ occurs if $d=1$, and $\|P\|=4 l+2$ in this case. This is the case identified in Theorem 1. Finally, suppose $l=2$ and $s=3$; then the maximal cancellation must occur among both the $t_{i}$ 's and the $u_{i}$ 's. Thus, $t_{2}=t_{1}+1$ and $t_{3}=t_{1}+1$ or $t_{2}+1$, and $\sum t_{i}=3 t_{1}+2$ or $3 t_{1}+3$. Similarly, $\sum u_{i}=3 u_{1}+2$ or $3 U_{1}+3$, so $t_{1}=u_{1}$, which is a contradiction. Thus, the only case which occurs is the one given in the first theorem. 


\section{REFERENCES}

1. M. Anshel and D. Goldfeld, Applications of the Hardy-Ramanujan partition theory to linear diophantine problems, J. Ramanujan Math. Soc. 3 (1) (1988), 97-110.

2. M. Anshel and R. Prener, On free products of finite abelian groups, Proc. Amer. Math. Soc. 34 (1972), 343-345.

3. M. N. Bleicher, A new algorithm for the expansion of Egyptian fractions, J. Number Theory 4 (1972), 342-382.

4. I. G. Connell, $A$ number theory problem concerning finite groups and rings, Canad. Math. Bull. 7 (1964), 23-34.

5. W. Magnus, A. Karrass, and D. Solitar, Combinatorial group theory: presentations of groups in terms of generators and relations, Dover Publications Inc., New York, 1976.

6. M. R. Schroeder, Number theory in science and communication, 2nd enlarged edition, Springer-Verlag, Berlin, Heidelberg, New York, and Tokyo, 1986.

7. B. L. Van Der Waerden, Science awakening, Oxford University Press, New York, 1961.

8. S. Wolfram, Mathematica ${ }^{T M}$ : a system for doing mathematics by computer, AddisonWesley, Reading, MA, 1988.

Department of Computer Sciences, The City College of the City University of New YORK, NEW YORK, NEW YORK 10031

Department of Mathematics, Columbia University, New York, New York 10027 